\title{
Designing Collaborative Healthcare Technology for the Acute Care Workflow
}

\author{
Michael J. Gonzales, Vanice C. Cheung, and Laurel D. Riek \\ Dept. of Computer Science and Eng. \\ University of Notre Dame \\ Notre Dame, IN 46556 \\ Email: \{mgonza14, vcheung, lriek\}@nd.edu
}

\begin{abstract}
Preventable medical errors in hospitals are the third leading cause of death in the United States. Many of these are caused by poor situational awareness, especially in acute care resuscitation scenarios. While a number of checklists and technological interventions have been developed to reduce cognitive load and improve situational awareness, these tools often do not fit the clinical workflow. To better understand the challenges faced by clinicians in acute care codes, we conducted a qualitative study with interprofessional clinicians at three regional hospitals. Our key findings are: Current documentation processes are inadequate (with information recorded on paper towels); reference guides can serve as fixation points, reducing rather than enhancing situational awareness; the physical environment imposes significant constraints on workflow; homegrown solutions may be used often to solve unstandardized processes; simulation scenarios do not match real-world practice. We present a number of considerations for collaborative healthcare technology design and discuss the implications of our findings on current work for the development of more effective interventions for acute care resuscitation scenarios.
\end{abstract}

Keywords-health informatics; workflow; acute care; resuscitation; computer-supported collaborative work; healthcare technology design; user-centered design

\section{INTRODUCTION}

Healthcare in the United States needs to be safer. As many as 400,000 people die annually in hospitals alone as a result of preventable medical errors (PMEs) [1]. PMEs are mistakes clinicians make that result from improper drug administration, diagnostic errors, and arise in situations of high cognitive load. Nationally, they are the third leading cause of death, with an estimated total cost of approximately $\$ 17$ billion per year [2].

PMEs may occur more frequently in resuscitation scenarios, or codes, in acute care environments, which include the adult and pediatric intensive care unit (ICU), emergency department (ED), and operating room (OR) [3]. Interprofessional clinicians generally work in these environments as teams. Clinical teams are burdened with a high cognitive load due to a reliance on collective memory and verbal communication in acute care [4], [5]. As a result, missteps and resource mismanagement can occur, especially among team members with varying experience and mental models [6], [7], [8].

PMEs can lead to a host of problems in acute care environments. In addition to patient death and adverse harm, they may also lead to errors in the documentation of events. This means clinicians may have either no record or an incorrect record of events that occurred while a patient was in their care. By law, this negligence is considered malpractice [9].

In order to avoid PMEs, clinical teams need to maintain sufficient situational awareness (SA), which is the ability to interpret information and make effective decisions in a situation. In acute care, team members need to be aware of one another, the patient, and the ongoing tasks around them. Maintaining SA is particularly challenging in acute care settings due to their chaotic and unpredictable nature [3].

Checklists are one type of intervention aimed at bridging this gap [4]. They are used commonly in the aviation industry, providing a systematic approach to tasks to avoid mistakes and memory lapses. Checklists can reduce cognitive load by breaking down complex processes for rapid responses to crises.

There are clear safety advantages to introducing welldesigned checklists into the clinical workspace [10], [11]. Haynes et. al [10] found that using checklists in the operating room reduced the overall death rate by nearly $50 \%$, and the overall complication rate decreased by $36 \%$. In addition, the use of checklists led to increased preventative measures (e.g. double-checking patient pain and medication administration) [12], [13]. Checklists are particularly useful for novices to use as memory aids for the effective execution of sequences that are too complex to hold in memory alone.

However, checklists are not a universal fix for cognitive load issues and there are several obstacles preventing their adoption. For example, some providers feel checklists undermine their claims to expertise and that they infantilize and conflict with the time-sensitive decision making process [14]. Poorly designed checklists may also create problems with information retrieval, causing clinicians to scan through dense text, tables, or across multiple pages [4]. Their overuse can also be dangerous, encouraging dependency and hampering the development of medical intuition from experience [11]. Furthermore, research exploring the efficacy of checklists in healthcare settings suggests some benefits may be dependent on the context, workflow, and practices of the institution [15].

Thus, before deploying interventions in the clinical workspace, we must first understand the context in which they are used. Even in similar procedures, the way hospitals respond to acute care codes (emergency situations) can differ drastically even with medical standards in place [16]. In order for tools to be effective against PMEs, we must consider more than just the context, but the clinical workflow and workspace [17]. 
In this paper, we present a set of considerations designers may need to take into account when developing collaborative healthcare technology for acute care resuscitation. Through a qualitative study with clinicians across three US hospitals, we found several interdepartmental and institutional differences that may impact the design of acute care interventions. For example, we found that in some institutions, documentation in acute care is captured on paper towels, spacing can be as limited as 100 square feet for an eleven person team, and that current reference guides distract from the situation at hand.

Our work is similar in approach to those focused on trauma units and ICUs [18], [19]. However, rather than an in-depth analysis of a particular hospital and department's workflow, we focus on the breadth of differences between multiple hospitals for resuscitation workflows. We discuss the problems we found, potential solutions, and future directions for more effective intervention design.

\section{BACKGROUND}

\section{A. Situational Awareness}

$\mathrm{SA}$ is "the perception of elements in the environment within a volume of time and space, the comprehension of their meaning, and the projection of their status in the near future." SA is composed of three stages: Perception (Level 1), Comprehension (Level 2), and Projection (Level 3) [20].

Perception consists of the perception of elements and critical factors in the environment. Comprehension relates to the meaning of those elements and integrating this information to understand how they impact goals. Finally, projection involves anticipating future events based on their comprehension of a situation and environmental elements.

SA is context dependent and my be influenced by the environment an individual is working in [21]. It also involves a person's experience and ability to anticipate future events, make appropriate decisions despite time constraints, and execute those decisions [22].

\section{B. Technology Interventions for Improved SA}

The fields of human factors and human-computer interaction have employed various means to augment clinical settings for teams [11], [23], [24], [25], [26], [27]. France et al. [28] found electronic whiteboard use reduces physician workload in an ED, resulting in fewer workflow interruptions. Others explored the use of shared mobile devices and large displays for teams working throughout a unit, showing that shared systems can improve team task coordination [23], [24].

Shared displays are one possible solution for clinicians to form a cohesive mental model of a situation, enabling improved coordination and information exchange [29]. These systems provide a co-located team simultaneous visual information access, and can be composed of various displays, including: large displays, projected displays, and mobile devices. In healthcare, shared displays enable clinicians to manage patient information, better attend to ongoing tasks, and view critical information. They have been applied as cognitive aids to support SA in a variety of ways [11], [23], [24], [27].

If we introduce technology to acute care domains, it must not interfere with a teams' ability to conduct their work.

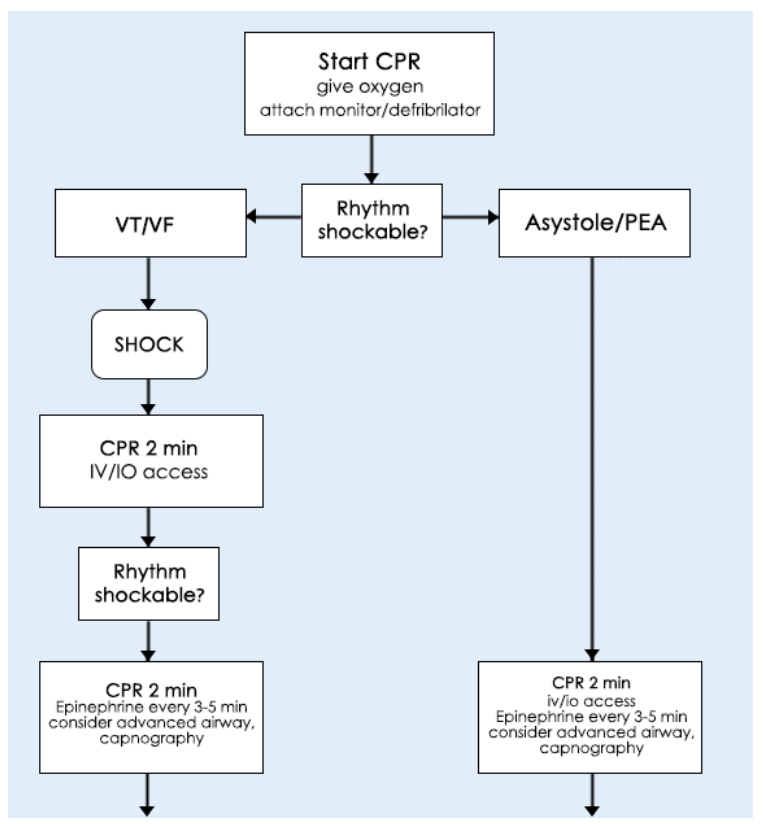

Fig. 1. A section of an ACLS diagram, without supplementary information

If technology creates more inconsistencies than paper does, it will lead to new problems and contribute to the field's resistance to new healthcare technology [30].

\section{Acute Care Algorithms and Codes}

Acute care is a branch of medicine that focuses on treatment for serious injuries [31]. It includes the ICU, pediatric ICU, and emergency department, among others. Clinicians work in teams in acute care codes, which often involve patient resuscitation.

Teams typically follow standardized algorithms for patients who require cardiac or respiratory resuscitation. Adult Cardiac Life Support (ACLS) [32] is one such algorithm for adults, while Pediatric Advanced Life Support (PALS) [33] is a set of algorithms used commonly for children. Both are evidencebased algorithms published by the American Heart Association (AHA), and are updated every five years. ACLS and PALS are commonly used in time-sensitive "code blue" situations, which result from either a respiratory or cardiac arrest.

Clinicians take an ACLS or PALS course to be certified to lead and participate in codes. The courses include written tests, descriptive videos of scenarios, and simulation components. The simulation component involves each clinician (regardless of profession) leading a simulated code. The patient in a simulated code is either a type of medical mannequin or an actor.

PALS reference guides are provided to each clinician who completes a PALS course. Each clinician working on a pediatric floor generally carries the guide with them. They contain diagrams similar to ACLS and other resuscitation scenarios, but for situations involving children.

Team leaders, the person responsible for directing the rest of the team, typically use reference guides for ACLS and PALS scenarios. An example of an ACLS algorithm is shown in Fig. 


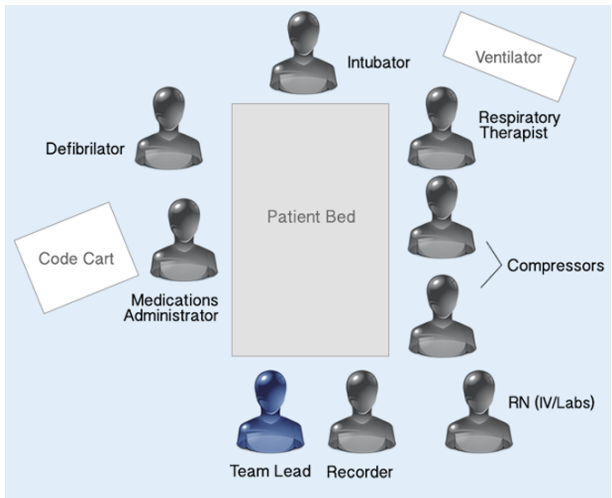

Team Leader: Dictates tasks to team. Typically an intensivist (person certified to lead acute care codes) or hospitalist (physician who primarily treats patients in the hospital).

Recorder: Documents all tasks performed during acute care codes, including timing and duration of tasks.

Respiratory Therapists: 1 - 2 people involved in stabilizing the patient's breathing, including intubation and ventilation.

Medication Administrator: Pharmacist or nurse responsible for administering drugs. Compressors: Person(s) responsible for chest compressions on the patient (CPR). Unit Assistant: Nurse who may be responsible to obtain supplies.

Phlebotomist: 1-2 people responsible for lab-related work.

Social Worker/Chaplain: Person responsible for communicating with family. May also be a nurse Ancillary Staff

Fig. 2. The general positions of each clinician during an ACLS and PALS code, along with a description of each person's role informed by our interviews.

1. The flowchart breaks down each step in the procedure with supplemental information displayed in a sidebar for reference.

Due to their time-sensitive nature, ACLS and PALS require a high-degree of team coordination and focus at all times. For example, the ACLS and PALS Pulseless Arrest algorithms require clinicians to rotate the person performing chest compressions (pushing down on the chest) on the patient every two minutes. It is the recorder's job to keep track of this, inform the compressors, and inform the person handling the defibrillator to perform a shock in between this change.

All of this information is documented in the hospital's code blue record, which is a form used to summarize everything that occurred in a code blue situation. Documentation is especially important, as it is the team's only form of evidence for what was actually done in a code [9]. A failure to accurately document a code may result in PMEs and potentially litigation.

Team sizes can range from five to twenty people. Fig. 2 shows the common roles and positions during code situations. These roles are assigned by a primary nurse as team members enter the room. Roles can change throughout a scenario based on the professions and expertise of those entering the room. As a result, team composition varies widely. At any given time, teams might consist of two to three intensivists, hospitalists, residents, and respiratory therapists, and up to four to six nurses. In non-critical floor units (e.g. the pediatric unit), the number of people can double according to our participants.

\section{Research Methods And Settings}

To understand the dynamics and problems being faced in acute care settings, we conducted a qualitative, multi-faceted study across three regional mid to large-sized hospitals in the United States. Our study consisted of semi-structured interviews and contextual inquiries with seven clinicians from different professions, including: education, nursing, emergency medicine, trauma care, and pediatric intensive care. We focused on acute care training, institutional differences, and environmental workflow. We also investigated the environmental constraints, equipment problems, spacing issues, and differences between training and real-life scenarios.

\section{A. Settings and Participants}

Our study participants included a PALS course instructor/ICU nurse, a senior nurse administrator, a trauma nurse, a hospitalist, two pediatric intensivists, and one emergency department physician. We chose these participants to offer us insight into differences in roles in acute care situations.

Our settings included two mid-large sized hospitals in the midwest (referred to as Midwestern Regional and Midwestern Children's for the remainder of the paper), and one midsized hospital (referred to as Northeastern Regional) in the northeastern US.

\section{B. Data Collection and Analysis}

We collected four kinds of data at each institution: interview data, artifact documents, sketches of the environment, and observational field notes. Field notes were collected for observed simulated situations, courses, and contextual inquiries. Data collection occurred over a period of five months. All interviews (approximately two, one-two hour interviews per clinician) were recorded. In total, our interview and observational data collection totaled approximately 40 hours, with about 12 hours of recorded data.

We transcribed and coded interview data using both inductive and deductive coding steps by two independent coders. Coders conducted an initial coding step to identify descriptive codes for each independent session with participants. Coders then developed a consistent coding scheme, and categorized codes into specific domains/taxonomies. Coders discussed and resolved any disagreements between codes after their analysis. The interview data had high inter-rater reliability $(k$-alpha $=$ 0.79 ), as calculated on a subset of the data.

Collected artifacts included the following documents: PALS training and course materials, PALS reference guides, ACLS course materials and reference guides, code blue paper documents, paper towel documentation, and home-grown spreadsheets used for patient evaluation. We coded and labeled artifact documents by their context of use and by location. We also labeled differences between artifacts from one hospital to the next, and compared these with standardized tools.

We took observational notes and created sketches of each room to document spacing limitations and constraints. We documented environmental factors such as equipment and team-member positioning within each room during ACLS and PALS codes. Sketches were later converted to digital form for analysis. We labeled equipment in the room based on our inquiries and gathered floor plans for space measurements. 
We also observed two PALS courses, which included nine, 15 minute simulations each at Midwest Regional Hospital, where each case was dictated by the instructor. We also observed ten, five minute "rapid recognition" simulated codes at Midwest Childrens Hospital. Rapid recognition mock codes involved a team leader calling a code for a patient, while having to identify their state using only the monitor and the patient's pulse, without any input from the instructor.

\section{KEY FINDINGS}

Our data revealed five key factors impacting clinical team workflow during acute care codes. First, we found that essential documentation practices suffer from inconsistencies, due to information capture on paper towels. Second, we found problems with reference guides creating fixation points for clinicians. Third, we found instances of workarounds used in attempts to solve SA and informational problems. Fourth, we observed the constraints imposed by space. Finally, we found striking differences between simulated and real-life codes.

\section{A. Documentation Practices}

Our participants described documentation practices as a common problem in acute care resuscitation codes. Officially, recording nurses should record tasks using either a code blue form or an EHR. However, while most codes start out this way, they are rarely used after the first few minutes.

Official documentation forms are rarely used because of the time-sensitivity of acute care codes. During a code, a recording nurse must simultaneously keep track of tasks performed, the time a CPR cycle has been given, the timing of a drug dose, and a narrative of events.

According to participants, EHRs and documentation forms are not properly designed for these types of situations. Instead, clinicians across all hospitals acknowledged that documentation tends to be taken on a napkin or paper towel (see Figure 3). As one physician put it, "Documentation is a big problem. [In] most hospitals, the code documentation takes place on a paper napkin. Everywhere I've ever worked, they'd grab a napkin and start documenting on it. We have a code blue form, but its usually filled out after the fact."

According to participants, the use of paper towels is a result of a form or EHR's inability to "keep up with everything going on." Documentation may start on one of these mediums first, but eventually breaks down. This may lead to SA problems for the recording nurse, leading to errors like incorrect or missing information. According to one nurse, "A lot of times [forms] will end up getting rewritten because you are jotting down information as you go, and you are missing pieces of information, and there are things that are written down on paper towels that you have to go back in and add. Sometimes there are issues with that, its just hard to capture everything in real time." Because the notes can be disjointed and illegible, problems arise due to a mismatch between what occurred and what is recorded or interpreted.

There are some beneficial affordances to the use of paper towels. Paper towels may act as temporary cognitive artifacts in certain instances and are quick and easy to access to write notes down [34]. They can be used as a quick way to store

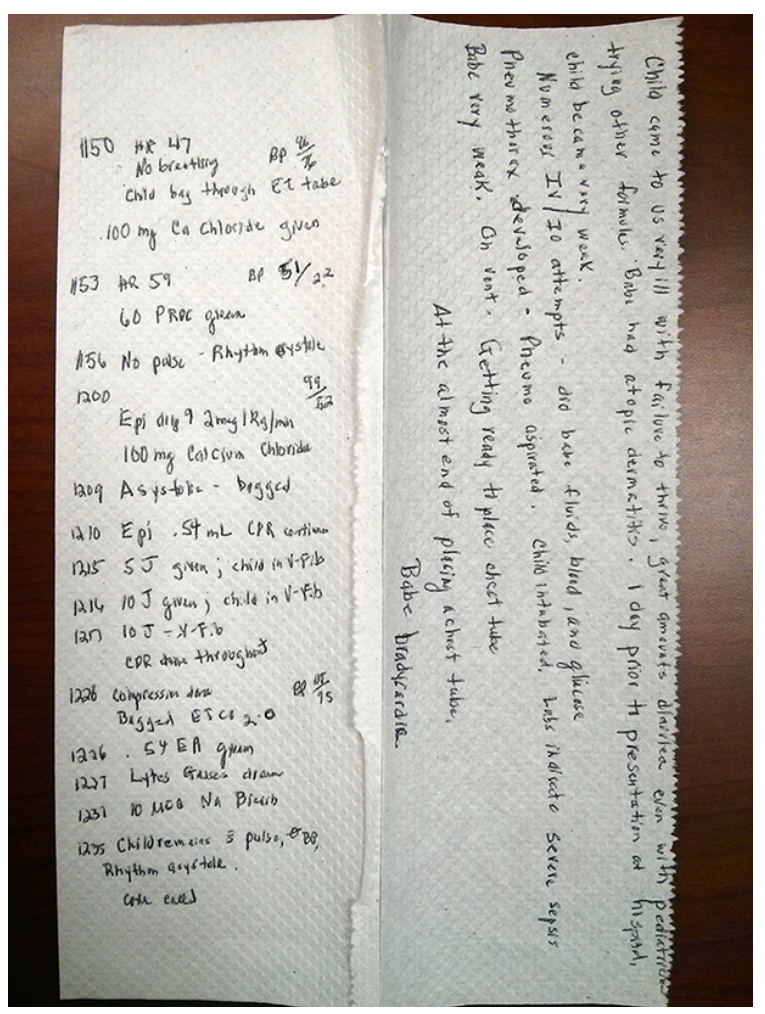

Fig. 3. A paper towel artifact from a recording nurse. Notes on the paper towel are later transferred to a code blue record or EHR.

information when official records or EHRs are not available or do not fit the time sensitive nature of a situation. However, it is because they are temporary aids that problems arise. Sequential notes may not always be recorded that way and key information may be lost in translation.

Thus, inconsistency remains a problem in codes due to an information mismatch. For example, a common issue was a chance of drug information being improperly interpreted when copying information to a code blue form. Furthermore, it becomes difficult to form a cohesive narrative of events when notes may be scribbled in different parts of the page, especially when compared to the level of detail a code blue form requires.

\section{B. Reference Guides}

We found problems in the overreliance of reference guides, which include the ACLS (see Fig. 1), and PALS reference cards. Reference guides are commmonly used by novice clinicians to act as cognitive aids for tasks during code situations. Team leaders may use a reference guide as a decision aid during code situations, whereas their teammates may use one to anticipate future events.

According to one physician, it is difficult to "process whatever they are putting on those [reference] cards by [the] brain of somebody who is running a code who doesn't usually." As a result, reference guides tend to be unintentional fixation points for novice clinicians during training and real-life scenarios. Instead of focusing on the patient's situation and their team, inexperienced clinicians may focus more on the guide. This disruption to SA may diminish the utility of aids designed for improving patient outcomes. 
One reason reference guides may serve as unintentional fixation points for clinicians is their size. An ACLS guide typically takes up an 8.5 " x 11 " page, and may include multiple diagrams on the same page. Thus, information is rather small on reference materials, causing clinicians to spend more time parsing text, figures, and keeping track of where they are. For example, the supplemental information on the ACLS guide has dense text in a list format along a side pane, which can lead to problems with information searching.

Similarly, the PALS reference card is a 23" $\mathrm{x} 14.5$ " six page fold out with six different treatment cases. The card contains drug information, a small Broselow tape, and supplemental information printed on both the front and back of each page. Generally, information is cluttered and contains lots of text within a small space. As one put it, "They aren't the most effective way of doing it. Partly size, partly just [because] it's hard to read when your attention is focused on the card when there's a code going on."

Generally, experienced physicians (like intensivists and hospitalists) do not use a reference guide and use memory for anticipating next steps during a code. However, we learned that a nurse is typically a first responder to a code and frequently uses guides whether or not they are leading. We observed this behavior in simulations where all participants used their reference guides. Clinicians typically had their head down reading over various parts of the guide to decide how to approach a patient scenario. Instructors commonly reminded participants to focus their attention on the patient and to use the guides as a reference aid only if needed. Interviews with instructors revealed this overreliance by novice clinicians occurs commonly in actual code situations. Thus, the guides may implicate patient safety due to their effect on SA and potential disruption to team performance.

\section{Cognitive Artifacts and Workarounds}

We found several cognitive artifacts and workarounds to common problems in the acute care environment. These included laminated cards and spreadsheets to calculate drug information based on a patient's weight and methods to manage people in the room. Clinicians employed these solutions as workarounds to help mitigate information look-up problems with patient records or the influx of people entering the scene.

Typically in emergency environments, an item called a standardized, proprietary tool called the Broselow tape is typically used in pediatric emergency resuscitation scenarios (see Fig. 4) [35]. The tape is a low tech tool designed to help clinicians save time for dosing calculations. Broselow tapes are used most commonly in the ED, rather than PICU, because of the lack of information on a patient. The tape is used as a quick assessment for medication dosages for children, and is based on estimates of height and weight. To aid clinicians visually, the ED has color-coded drawers on their code carts that directly mapped to doses of medication for children in a particular height range. This correlation can help clinicians easily identify necessary doses in time-critical situations.

In the PICU and pediatric unit of Midwestern Regional, a homegrown spreadsheet made by a nurse is used instead of a Broselow tape. A small laminated card is used at Midwest Children's in the same units. The main problems with these

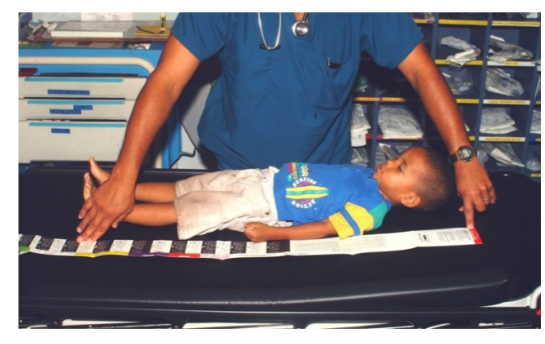

A

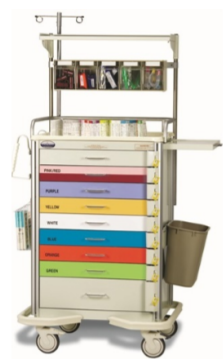

Fig. 4. A) A photo of a Broselow tape being used on a child. B) A code cart colored to match the Broselow Tape.

solutions are that they manually need to be updated to match recent findings in the literature and still suffer from many of the same information searching problems as reference guides. Because there is no standardized sheet for the computation of drug dosages, it is up to each unit or the hospital technology already put in place to solve medication look-up problems.

Nurses at Midwestern Regional preferred to use their own tool because it summarized key doses for a patient. However, the sheet itself has relatively hard to find text and tables, possibly affecting SA. The tool is used by entering a child patient's weight on a computer, which calculates dosages for the patient, and is then printed and kept in a file at the patient's bedside. Thus, the tool serves as a more accurate reference form for the Broselow tape without the quick access. Because this solution was developed in-house, it is possible that clinicians have a sense of ownership of the tool, known as the IKEA effect [36], [37].

At Midwestern Children's, clinicians use the patient's EHR to determine medication dosages. However, one key challenge with this process is time. A clinician may not be able to log in to a system easily to look up information. Furthermore, the person performing the look-up (likely the recording nurse) will have to relay this information to the person drawing up medication. This process can suffer from possible information loss due to the influx of people and noise, leading to other workarounds. For example, clinicians at Northeastern Regional resorted to color-coded tags assigned by the primary nurse as a way to organize people coming into the room during an ACLS or PALS code. The solution aims to prevent confusion from role assignments and to manage unnecessary personnel.

Since Northeastern Regional is a smaller hospital, a colorcoded form of personnel management worked well. In contrast, the other hospitals have a person relay instructions while the primary nurse handles incoming personnel mentally. For example, the ED and pediatric units of Midwestern Regional used code time-outs, which are situations where everyone stops to listen and receive instructions from a team leader. This allows everyone to get a clear picture of the patient's condition while incoming team members are briefed at the door.

Participants at Midwestern Children's do not use these methods in their own codes due to the sheer quantity of people and noise. In particular, Midwestern Children's general pediatric unit may have upwards of 20 people moving in and out of a room when a code occurs. This process must be managed by the primary nurse. 


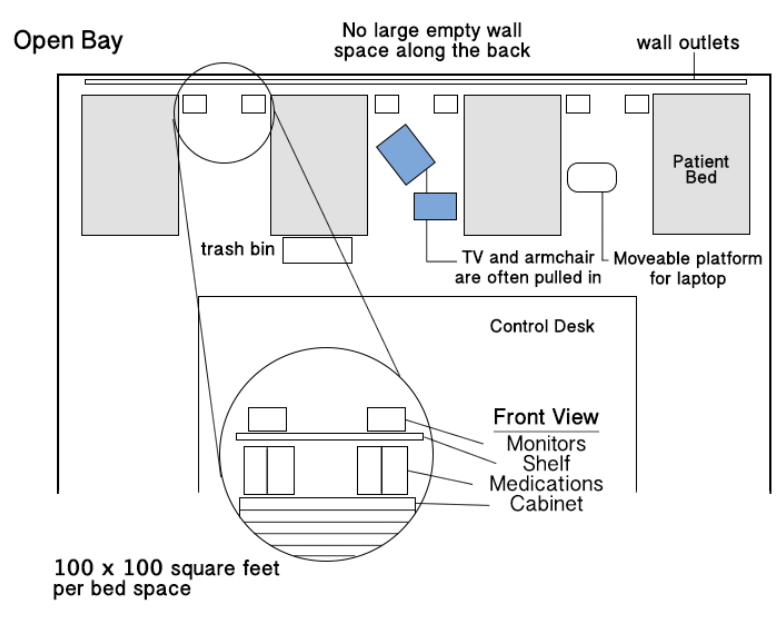

Fig. 5. The open bay at Midwestern Regional was approximately 100 sq. feet. With team sizes as large as 11 and the presence of equipment, it is challenging for clinicians to perform even basic duties, let alone maintain SA.

\section{Space and Movement Constraints}

Space constraints are another major problem faced in acute care since team sizes can be so large. Room dimensions were as small as 190 square feet in the non-pediatric wings, which occasionally have acute care codes. Within the PICU, Midwestern Regional had even tighter constraints, with four beds separated by only curtains in an open bay with as little as 100 square feet per bed. The open bay is shown in Fig. 5 . In contrast, Midwestern Childrens did not frequently encounter any space constraints, with the exception of the pediatric unit when 20 people were involved in a code in a 450 sq. ft. room.

With an influx of new people and equipment constantly being added during a code, the space around the patient becomes an critical factor in smaller environments. Movement constraints affect clinicians' access to equipment if others are in the way. They may also directly interfere with each others' duties, which may lead to adverse events or errors. Visitors in the room may further limit available space.

To accommodate for spacing constraints, visitors and equipment are sometimes moved outside of the room so a team can resuscitate a patient. A nurse or social worker typically tends to the family's questions and needs while the rest of the team tends to the patient. This serves the purpose of preventing visitors from diverting the team's attention.

While generally we can assume that each hospital has differences and limitations in space-wise, there are several factors that may impact future intervention design. At Midwestern Regional, clinicians had trouble with equipment at the patient's bedside due to the available outlets and their layout. Clinicians plug in devices in a specific order to leave enough outlets available for other critical equipment. A misstep in this process could lead to patient safety problems if a device needs to be disconnected and moved. This may disrupt the team's workflow, SA, and potentially impact resuscitation efforts.

While wall space is generally unused in acute care codes, it is still rather limited. Wall space is generally occupied by equipment, notices, paneling, and large whiteboards used for routine check-ups. In some cases, curtains may serve as the only walls for patient beds. While wall space may not directly impact a clinician's SA, the lack of it also implies the inability for a team of clinicians to share information in a large open area so everyone can gain information about a situation.

\section{E. Differences Between Simulation and Reality}

There are a number of differences between simulation scenarios and the real world. These differences are important, because the training clinicians receive at each institution might vary, even for the same procedures. While AHA guidelines for ACLS and PALS are standardized, the tools and environments employed by each institution are not. New types of interventions will also need to be adaptable to this variance, as clinicians will need to start using new interventions in training.

The most important problem we found with simulations was that participants may not reflect the team composition and size in real-life scenarios. Teams may not be interprofessional, as they are in the real world, but rather single discipline teams. Furthermore, clinicians' role assignments in simulations may not match their duties in real-life scenarios.

In the simulations we observed, we found participants would typically call on others of the same profession. Simulations provide this flexibility, despite an instructor's advice, for clinicians to choose their team members for certain simulations. However, this is not reflective of real life. Team members must all work cohesively as a whole to stabilize a patient, amid ongoing noise, people, and chaos.

Furthermore, team sizes vary widely between simulations and real life. In courses, teams are composed of as little as five people, a severe mismatch between real-life situations that have as many as 20 . Thus, novice clinicians are inexperienced in working in large real world teams, and therefore may suffer from stress, cognitive overload, and therefore impede the functioning of their team as a whole.

We also found that in some instances, simulations may take place in a classroom or conference room, which is not reflective of real world constraints. This means a clinician may be unprepared for the constraints faced in real world situations, and can further hinder other team members' duties.

Simulations are also "pause" friendly, meaning it is possible for the team leader to stop and ask questions or take time to use tools. According to instructors of the courses, the learning and thought process are the most important aspects of the simulations. We observed a similar approach in the "rapid recognition" scenarios, albeit with less instructor interaction. Unfortunately, the real world does not offer this flexibility. Thus, clinical learners must attune themselves to the constraints and time-sensitive nature of real world scenarios.

\section{DISCUSSION AND IMPLICATIONS}

Designing healthcare technology for acute care situations is a challenging task. We not only need to take into account human and environmental factors, but also clinicians' workflow. Each hospital, and even departments within the same hospital may have its own set of constraints and be affected by different sets of problems. Thus, if we aim to improve the acute care workflow, and reduce PMEs, it is important that we do so without introducing equipment that creates new hazards and errors. 


\section{A. Augmenting Documentation Practices}

One of the most striking problems we uncovered was the way information is recorded during acute care code situations. Because official code blue records and digital EHRs suffer from the capability to keep up with a situation, clinicians at all institutions we worked with acknowledged the use of a napkin or paper towel for recording tasks.

This is a huge problem that has serious implications for not only the patient, but clinicians themselves. Missteps in documentation may be taken as factual representations of what actually happened, and a clinician may be wrongly accused of malpractice if the record is drawn up in court for a patient death [9]. While it is unlikely we can replace paper entirely (work by [27] explored this potential using digital handwriting technology), it is possible we may be able to augment documentation practices.

One possible way we can augment documentation practice is the use of an application that can track the timing, ordering, and completion of tasks during code situations. For example, a clinician can click a button on an app to start timers for CPR and Epinephrine. When the timer for each task reaches its limit, an indicator can blink so the recorder can let the rest of the team know a new action must be performed. This can help reduce the attentional demands of tracking multiple tasks so the recorder can focus on the narrative of events.

Participants reacted positively to the idea of a documentation utility with time-tracking features to aid recording nurses. One clinician noted that,"Being able to click a button ... that medication was given so that you could capture things more real time ... would be helpful." Tools like this may not replace the culture of using the paper towel, but may help reduce the divided attention recording nurses face in documenting tasks.

\section{B. Designing to Support Collaboration in Constrained Spaces}

One of the frequent problems we encountered was the overreliance on reference guides by novice clinicians. Instructors noted that clinicians tend to focus too much on the guides rather than the situation at hand. A technological intervention such as a shared display might help address this problem, by enabling clinicians to keep their heads up during a situation, rather than concentrating on a card.

However, when we consider new tool design, we must be mindful of the constraints already faced by clinicians. The spatial dimensions of the room will also affect the interaction modality and display type. For example, previous work employed shared displays as a way for clinicians to keep tabs of a situation [11]. In this work, a large monitor was attached to a code cart with the idea that team members could view information, while a clinician used a tablet to control the system. Based on the findings of our work, a solution such as this may lead to more problems by 1) having the leader focus on a tablet computer much like a reference card, 2) imposing additional environmental constraints by drawing cords across the room for the monitor and 3) taking up additional outlets for critical equipment.

Thus, new interventions should either use existing equipment advantageously, or be designed so as not to introduce new hazards. For example, patient rooms with TVs can be used as shared displays for teams. This is appealing because rooms may already have TVs built into the wall to be unobtrusive during codes. Alternatively, projected displays are another viable option if wall space is available. Projected displays are adjustable and portable enough to accommodate the movement of clinicians. In addition, newer pico-projectors also have internal batteries, reducing hazards caused by power cords.

Interest in head-mounted optical displays like Google Glass for healthcare is on the rise as well [38]. The device can either be used by the team leader only, or by multiple team members with devices allocated based on their role during a code. Ensuring that clinicians' tasks link properly and that they maintain SA without distractions from prompts still requires evaluation.

Finally, large touch-screen laptops and dual-screen tablets might be well suited for ICU and floor units without wall space. Display sizes range up to 17" screen sizes, enabling both the team leader, recorder, and members in the immediate vicinity viewable access to screens. Dual-screen laptops may enable groups working on either side of a crash cart access to information on a screen. Further exploration into alternative interaction modalities may yield additional options.

\section{Institutional Culture}

The culture of an institution will also impact design. Each hospital will have their own process and procedures on how specific activities are done, and may use home-grown methods in attempts to solve issues. Thus, when we enter each space as designers, we must ensure we understand the specific procedures and practices of each institution such as not to negatively impact workflow and create more errors. How do clinicians in each space handle information dissemination? What home-grown tools are used and what benefits, if any, do they offer over other tools? How can our tools augment the real world and clinical training?

Clinicians might exhibit resistance to the removal of their own solutions to problems, or those they are unfamiliar with. This sense of ownership, the IKEA effect [36], might prevent the adoption of new types of tools. Thus, it is important we design adaptable frameworks to accommodate the constraints and practices of institutions, and tailor tools through co-design strategies so that ownership transfers. This must extend to applications for both the simulation and real-world such as not to disrupt clinical workflow.

In addition, the primary and secondary users of interventions must be well established for acute care [39]. A primary user would be the individual actually controlling an intervention, while secondary users are those using the system in a shared manner. In particular, we must understand each person's role in a situation, and take cognitive resources into account when we introduce new interventions to the workspace.

Finally, the impact of others in the room is another crucial component for designers. If we design new interventions for acute care, we must consider how their addition to the clinical workspace might affect providers' workflow. If parents or family can see the set of steps clinicians follow and observe skipped steps along the way, they may interrupt providers during critical decision points. 


\section{LIMITATIONS AND FUTURE WORK}

We acknowledge that there are several limitations to this work. First, our study represents a subset of clinicians from three hospitals in the US, which do not represent every hospital, their departments, or situations. Second, this work does not reflect all aspects of acute care, and thus, further work is required to establish additional constraints. Despite these limitations, however, we believe this work represents an important contribution in collaborative healthcare technology design for codes. Moving forward, we will utilize this work as a basis for the design of a collaborative aid for acute care resuscitation codes. Through user-centered design, we are developing and will evaluate a shared cognitive aid so that clinical teams can reference during codes.

\section{REFERENCES}

[1] J. James, "A New, Evidence-based Estimate of Patient Harms Associated with Hospital Care." Journal of patient safety, vol. 9, no. 3, 2013.

[2] C. Andel, S. L. Davidow, M. Hollander, and D. A. Moreno, "The economics of health care quality and medical errors," Journal of health care finance, vol. 39, no. 1, 2012.

[3] L. T. Kohn, J. Corrigan, M. Donaldson et al., To err is human: Building a safer health system. Committee on Health Care in America. Institute of Medicine. National Academy Press, 1999.

[4] A. Gawande, The Checklist Manifesto. Penguin, 2010.

[5] A. Sarcevic, I. Marsic, M. E. Lesk, and R. S. Burd, "Transactive memory in trauma resuscitation," in Proceedings of the 2008 ACM conference on Computer supported cooperative work. ACM, 2008.

[6] J. M. Davies, "Team communication in the operating room." Acta anaesthesiologica Scandinavica, vol. 49, no. 7, 2005.

[7] L. Lingard, "Communication failures in the operating room: an observational classification of recurrent types and effects," Quality and Safety in Health Care, vol. 13, no. 5, Oct. 2004.

[8] R. Santos and et al., "Characterization of non-technical skills in paediatric cardiac surgery: communication patterns." European journal of cardio-thoracic surgery, vol. 41, no. 5, 2012.

[9] J. M. Lyons III, J. A. Martinez, and J. P. O'Leary, "Medical malpractice matters: medical record m \& ms," J SURG EDUC, vol. 66, no. 2, 2009.

[10] A. B. Haynes, T. G. Weiser, W. R. Berry et al., "A surgical safety checklist to reduce morbidity and mortality in a global population." The New England journal of medicine, vol. 360, no. 5, 2009.

[11] L. Wu, J. Cirimele, K. Leach, S. Card, L. Chu, T. K. Harrison, and S. R. Klemmer, "Supporting crisis response with dynamic procedure aids," in Proceedings of the 2014 Conference on Designing Interactive Systems, ser. DIS '14. ACM, 2014.

[12] C. Cheney and J. W. Ramsdell, "Effect of medical records' checklists on implementation of periodic health measures," $A M J M E D$, vol. 83, no. $1,1987$.

[13] P. Pronovost and D. Needham, "An intervention to decrease catheterrelated bloodstream infections in the ICU," NEJM, 2006

[14] C. L. Bosk, M. Dixon-Woods, C. A. Goeschel, and P. J. Pronovost, "Reality check for checklists," The Lancet, vol. 374, no. 9688, 2009.

[15] D. R. Urbach, A. Govindarajan, R. Saskin, A. S. Wilton, and N. N. Baxter, "Introduction of surgical safety checklists in ontario, canada," New England Journal of Medicine, vol. 370, no. 11, 2014.

[16] J. E. Wennberg, "Dealing with medical practice variations: a proposal for action," Health Affairs, vol. 3, no. 2, 1984.

[17] L. Poissant, J. Pereira, R. Tamblyn, and Y. Kawasumi, "The impact of electronic health records on time efficiency of physicians and nurses: a systematic review," J AM MED INFORM ASSN, vol. 12, no. 5, 2005.

[18] S. Malhotra, D. Jordan, E. Shortliffe, and V. L. Patel, "Workflow modeling in critical care: piecing together your own puzzle," Journal of biomedical informatics, vol. 40, no. 2, pp. 81-92, 2007.

[19] M. Vankipuram, V. Ghaemmaghami, and V. L. Patel, "Adaptive behaviors of experts in following standard protocol in trauma management: implications for developing flexible guidelines," in AMIA Annual
Symposium Proceedings, vol. 2012. American Medical Informatics Association, 2012, p. 1412.

[20] M. R. Endsley, "Toward a theory of situation awareness in dynamic systems," Human Factors: The Journal of the Human Factors and Ergonomics Society, vol. 37, no. 1, 1995.

[21] N. B. Sarter and D. D. Woods, "Situation awareness: A critical but ill-defined phenomenon," The International Journal of Aviation Psychology, vol. 1, no. 1, pp. 45-57, 1991.

[22] L. Carroll, "Desperately seeking sa," DTIC Document, Tech. Rep., 1992.

[23] C. Bossen and L. W. K. r. Jensen, "Implications of Shared Interactive Displays for Work at a Surgery Ward: Coordination, Articulation Work and Context-Awareness," 2008 21st IEEE International Symposium on Computer-Based Medical Systems, 2008.

[24] J. Favela, M. Rodriguez, A. Preciado, and V. Gonzalez, "Integrating context-aware public displays into a mobile hospital information system," IEEE T INF TECHNOL B, vol. 8, no. 3, 2004.

[25] J. Cirimele, L. Wu, K. Leach, S. Card, T. K. Harrison, L. Chu, and S. R. Klemmer, "Rapidread: Step-at-a-glance crisis checklists," in Proceedings of the 2014 IEEE/ACM Conference on Pervasive Systems in Healthcare, ser. PervasiveHealth '14, 2014.

[26] A. Parush, C. Kramer, T. Foster-Hunt, K. Momtahan, A. Hunter, and B. Sohmer, "Communication and team situation awareness in the or: Implications for augmentative information display," Journal of biomedical informatics, vol. 44, no. 3, 2011.

[27] A. Sarcevic and N. Weibel, "A paper-digital interface for information capture and display in time-critical medical work," in Proceedings of the 2012 IEEE/ACM Conference on Pervasive Systems in Healthcare, ser. PervasiveHealth '12, 2012.

[28] D. J. France, S. Levin, R. Hemphill, K. Chen, D. Rickard, R. Makowski, I. Jones, and D. Aronsky, "Emergency physicians behaviors and workload in the presence of an electronic whiteboard," International journal of medical informatics, vol. 74, no. 10, 2005.

[29] C. A. Bolstad and M. R. Endsley, "The effect of task load and shared displays on team situation awareness," in Proceedings of the Human Factors and Ergonomics Society Annual Meeting, vol. 44, no. 1. SAGE Publications, 2000, pp. 189-192.

[30] A. Bhattacherjee and N. Hikmet, "Physicians' resistance toward healthcare information technology: a theoretical model and empirical test," European Journal of Information Systems, vol. 16, no. 6, 2007.

[31] P. J. Pronovost, T. Nolan, S. Zeger, M. Miller, and H. Rubin, "How can clinicians measure safety and quality in acute care?" The Lancet, vol. 363, no. 9414, 2004.

[32] R. Neumar and et al., "Part 8: Adult advanced cardiovascular life support 2010 american heart association guidelines for cardiopulmonary resuscitation and emergency cardiovascular care," Circulation, vol. 122, no. 18 suppl 3, 2010.

[33] M. E. Kleinman and et al., "Pediatric advanced life support: 2010 AHA Guidelines for Cardiopulmonary Resuscitation and Emergency Cardiovascular Care." Pediatrics, vol. 126, no. 5, 2010.

[34] M. Hardey, S. Payne, and P. Coleman, "scraps: hidden nursing information and its influence on the delivery of care," Journal of Advanced Nursing, vol. 32, no. 1, pp. 208-214, 2000.

[35] D. S. Lubitz, J. S. Seidel, L. Chameides, R. C. Luten, A. L. Zaritsky, and F. W. Campbell, "A rapid method for estimating weight and resuscitation drug dosages from length in the pediatric age group," Annals of emergency medicine, vol. 17, no. 6, pp. 576-581, 1988.

[36] M. Norton, D. Mochon, and D. Ariely, "The'ikea effect': When labor leads to love. [harvard business school marketing unit working paper no. 11-091]," SSRN eLibrary, vol. 34, 2011.

[37] D. Mochon, M. I. Norton, and D. Ariely, "Bolstering and restoring feelings of competence via the ikea effect," International Journal of Research in Marketing, vol. 29, no. 4, pp. 363-369, 2012.

[38] O. J. Muensterer, M. Lacher, C. Zoeller, M. Bronstein, and J. Kübler, "Google glass in pediatric surgery: An exploratory study," International Journal of Surgery, vol. 12, no. 4, pp. 281-289, 2014.

[39] O. Alsos and D. Svans, "Designing for the secondary user experience," in Human-Computer Interaction INTERACT 2011, ser. Lecture Notes in Computer Science. Springer Berlin Heidelberg, 2011, vol. 6949. 